\title{
Isolation and Identification of Enterobacteriaceae from Patients with Community Acquired Urinary Tract Infection
}

\author{
Abd Elrahman Mustafa Abd Elrahman Osman *, Shingray Osman Hashim, \\ Mohammed Abdall Musa, Omer Mohammed Tahir
}

Medical Laboratory Sciences Division, Port Sudan Ahlia College, Port Sudan, Sudan

Email address:

abdo_rahman96@hotmail.com (A. E. M. A. E. Osman)

*Corresponding author

To cite this article:

Abd Elrahman Mustafa Abd Elrahman Osman, Shingray Osman Hashim, Mohammed Abdall Musa, Omer MohammedTahir. Isolation and Identification of Enterobacteriaceae from Patients withCommunity Acquired Urinary Tract Infection. American Journal of Health Research. Vol. 6, No. 1, 2018, pp. 25-31. doi: 10.11648/j.ajhr.20180601.14

Received: October 28, 2017; Accepted: December 4, 2017; Published: January 22, 2018

\begin{abstract}
The study was carried out in red sea state during the period from November 2013 to March 2014 to investigate the Enterobacteriaceae from patients suffering from community-acquired urinary tract infections and then do sensitivity test for each of isolates. In this study out of 100 specimens 52 Enterobacteriaceae species were isolated from different clinics and hospitals in Port Sudan city. The specimens werecultured on CLED media ( cystinelactose electrolyte deficiency). identification was done by gram's stain and convential biochemical reactions. Then the anti-microbial sensitivity tests were done as the follows: (Ampicillin-Sulbactam "AS", Co. trimoxazole "BA", Ceftizoxime "CI", Chloramphenicol "CH", Cephalaxin "PR", Tetracycline "TE", Ciprofloxacin "CP", Amikacin "AK", Sparfloxacin "SC", Gatifloxacin "GF", Norflaxcin "NX) by Kirby-Bauer disc diffusion method. The study revealed that the most part of strains are sensitive to Chloramphenicol andAmikacin and resistant to Ampicillin -Sulpactam (AS). The identified Enterobacteriaceae were as follows; Escherichia coli 34 (65\%), Klebsiella pneumonia 10 (19\%), Klebsiella oxytoca 3(6\%), Salmonella Para A 3 (6\%), proteus mirabilis 1 (2\%), Citrobacter 1 (2\%).
\end{abstract}

Keywords: Enterobacteriaceae, Urinary Tract Infections, Escherichia Coli, Red Sea State

\section{Introduction}

Urinary tract infection represents one of the commonest bacterial infections. The Enterobacteriaceae are the most frequent pathogen detected, causing most of the urinary tract infection, Enterobacteriaceae are a group of largest, most heterogeneous collection of medically important gram -ve bacilli, being found worldwide in soil, water and are part of the normal florain the intestine of most animal and human this family include many genera such as(Escherichia. Coli, Klebsiella, salmonella ...etc). These days the main cause for concern among Enterobacteriaceae especially that cause community acquired infection is Escherichia coli. [1].

A urinary tract infection (UTI) is a condition in which one or more parts of the urinary system (the kidneys, bladder, and urethra) become infected. Although some cases of UTI are due to fungus or a virus, most are caused by one of several types of bacteria most cases of UTIs are caused by bacteria that typically multiply at the opening of the urethra and travel up to the bladder. Much less often, bacteria spread to the kidney from the bloodstream [1].

The urinary system helps maintain proper water and salt balance throughout the body and also expels urine from the body The symptoms of urinary tract infections result from the presence and growth of bacteria or other microorganisms in the urinary tract. The urinary tract is normally a sterile environment. The bacteria, in some cases, can get flushed up into the kidneys and therefore can cause a kidney infection. Both kidney and bladder infections are more common in women because their urethras are shorter than men, making it easier for organisms to get from outside into the bladder. Most typically, a woman develops a UTI if she has been 
sexually active, (hence the moniker "honeymoon cystitis"), or has been careless with her hygiene habits (for example, wiping from back to front after a bowel movement) [2].

Early recognition of bacteraemic UTI and prompt, appropriate treatment are critical in reducing the mortality. A diagnosis of a urinary tract infection can easily be missed or delayed in the elderly. This is because some symptoms, such as fatigueand weakness, may not be noticed or might be associated with aging, Also the extensive and inappropriate use of antimicrobial agents has invariably resulted in the development of antibiotic resistance which, in recent years, has become a major problem worldwidefamily physicians can provide empirical treatment without the benefit of a pretherapy urine culture. the extensive and inappropriate use of antimicrobial agents has invariably resulted in the development of antibiotic resistance which, in recent years, has become a major problem worldwide. for example Quinolones which are one of the most widely used antibiotics in the community for the treatment of UTI, the unfortunate excessive use of this agent that has led to a considerable and worrying increase in the rate of $E$. coli resistantisolates in many countries, and this is just a start because as more patients use antimicrobial drugs without pre-therapy cultureas more resistant bacteria will gain. [2].

Urologists have tended to ignore the clinical importance and urologic realities of community-acquired urinary tract infections (UTIs) despite their significant prevalence, cost, morbidity, and increasing management problems. This is primarily because of our perception that uncomplicated UTIs are common but not a serious problem (patients do not die from uncomplicated UTIs), easy to diagnose (simple midstream urine culture), and simple to treat (short course of antibiotics). Nevertheless, data on increasing prevalence, cost, morbidity, antibiotic resistance, recurrence, and relapse suggest that the urological community needs to have another look at community-acquired UTIs. [3].

This study aimed to determine the frequency of isolated Enterobacteriaceae. and antimicrobial susceptibility of uropathogens in culture-positive community-acquired UTIs over a 3 months period, community-acquired UTIs over a four months periodin order to. Understanding the pathogenesis of UTI which may lead to better methods of prevention and treatment. [3].

\section{Materials and Method}

\subsection{Study Approach}

Study approach is to identify and isolate the possible causative organisms and the characterization of isolated strains using bio typing and anti bio gram.

\subsubsection{Study Type and Design}

Cross-sectional descriptive study.

\subsubsection{Study Area}

The study area is different hospitals and clinics in Red Sea state.

\subsubsection{Study Period}

During the period from November 2013 to March 2014

\subsection{Methodology}

\subsubsection{Sample Size and Collection}

According to standard method all specimen were examine to detect, and identify pathogens or their products using: microscopic examination of specimen to detect their motility, morphology and staining reaction. Also culture techniques to isolate pathogens in pure form and to identify them then test their antibiotic sensitivity and biochemical.

\section{a. Sampling}

Samples for community acquired UTI to investigate and diagnosis of microbial diseases (urine specimen).

\section{b. Urine Specimens (100 Samples)}

Urine specimens were collected in case of urinary tract infection as following. A mid stream urine is obtained in a sterile container after cleaning the external genitalia with tap water and drying. Samples should reach the laboratory within one hour after voiding or kept refrigerated at $4 c^{\circ}$ to avoid multiplication of bacteria in urine then we do appropriate test to identify and isolate the causative organisms.

\subsubsection{Cultivation of Specimens}

Urine specimens were inoculated onto CLED medium by using sterile loop, then incubated aerobically at $37 \mathrm{c}^{\circ}$ over night.

\subsubsection{Examination of Bacterial Growth}

The primary culture on CLED medium that showed significant growth was examined for fermentation. The morphological character, size, shape, colour were observed and recorded.

\subsubsection{Interpretation of Culture Growth}

The culture growth obtained was interpreted as significant $\left(>10^{5} \mathrm{CFU} / \mathrm{ml}\right)$. Cultured of less than $(103 \mathrm{CFU} / \mathrm{ml})$ of urine was considered insignificant, while culture with no growth were considered negative. Significant cultures were further investigated.

\subsubsection{Purification of Bacterial Growth}

The isolates were streaked onto Nutrient agar and incubated overnight at $37^{\circ} \mathrm{C}$. The resultant growth was checked for purity and stored in Bijou bottle for further investigation.

\subsubsection{Identification of the Isolated Bacteria}

\section{Colonial Morphology}

Colonial characteristic were observed on CLED medium after overnight incubation isolated organisms were growth on CLED medium.

\section{Biochemical Tests (Conventional Test)}

These are called biochemical tests because they are tests which identifythebacteria on the basis of the presence of certain enzymes and other biochemicalproperties. 


\section{Kliglar Iron Agar}

this medium was originally designed as a multi-test medium. It provides a low degree of sensitivity for $\mathrm{H} 2 \mathrm{~S}$ production (often required when differentiating members of the (Enterobacteriaceae). The medium is now used principally as a standard test for $\mathrm{H}_{2} \mathrm{~S}$. One disadvantage of multitest media is that chemical interaction-in this case acid production from fermentable sucrose-may inhibit blackening of the iron indicator. Some Citrobacter and Proteus species have this ability. KIA agar should be used in conjunction with a urease test to eliminate Proteus spp when screening for Salmonellae.

\section{Citrate Utilization Test}

This is for the ability of on organism to utilize citrate as the sole carbon and energy source forgrowth and an ammonium salt as sole source of nitrogen.

\section{Indole Test}

This test demonstrates the ability of certain bacteria to decompose the amino acid tryptophan toindole, which accumulates in the medium. Indole is then tested for by a colorimetric reactionwith p- dimethylaminobenzaldehyde. Add $0.5 \mathrm{ml}$ kovacs reagent to see result.

\section{Urease Test}

Bacteria, particularly those growing naturally in an environment exposed tourine, may decomposeureaby means of the enzyme urease The occurrence of this enzyme can be tested for by growingthe organism in thepresence of urea and testing for alkali production by means of a suitable PHindicator. An alternative method is to test for the production of ammonia from urea by meansNesslers reagent.

\section{Motility Test}

In semi solid agar media, motile bacteria (Swarm) and give a diffuse spreading growth that iseasily recognized by the naked eye. Motility may thus be detected more easily than by themicroscopical method.

\section{AntimicrobialSensitivity Test}

All isolated microorganisms were subjected to antimicrobial sensitivity test using ModifiedKirby-Bauer disc diffusion method [10].

\section{Results}

One hundred urine specimen were collected from patients suffering from UTI during the period from November 2013 to march 2014. Out of 100 patients (52\%) were positive, while (48\%) were negative. Among the positive growth 4 of them were NLF organisms (8\%), and $48(92 \%)$ were L. F organisms (Table 1).

The most frequent patients were female (75\%), 38 of them were positive cultureand the male were less frequent $(25 \%)$ only 14 of them were positive culture(Figure 1).

The isolated Enterobacteriaceae were as followed: Escherichia coli 34 (65.4\%), Klebsiella pneumonia 10 (19.2\%), Klebsiella oxytoca 3(5.7\%), Salmonella Para A 3 (5.7\%), proteus mirabilis 1 (2\%), Citrobacter 1 (2\%) (Figure 2).

For female patientsthe age ranged from (one year to 10 years) one patient showedpositive culture, 4 urine culture were positive from the ages (11 to 20 years). Seven urine culture were positive from the ages of (21 to 30 years). From the age of 31 to 40 years old 13 of them were positive urine culture and this is the most predominant ages infected with Enterobacteriaceae, from (41 years old to 50 years) 9 patients were positive urine culture and more than (50 years) only 4 patients were positive urine culture (Figure 3 ). For male patients the ages ranged from (one year to 10 years)one patient was positive urine culture, from the ages of (21 to 30 years) 4 patients were positive for urine culture, from (41 years to 50 years) 4 patientswere positive for urine culture. More than (50 years) represent the most frequent infection in male (Figure 4).

The result of antimicrobial sensitivity testsrevealed in (Table 2) and (Figure 5).

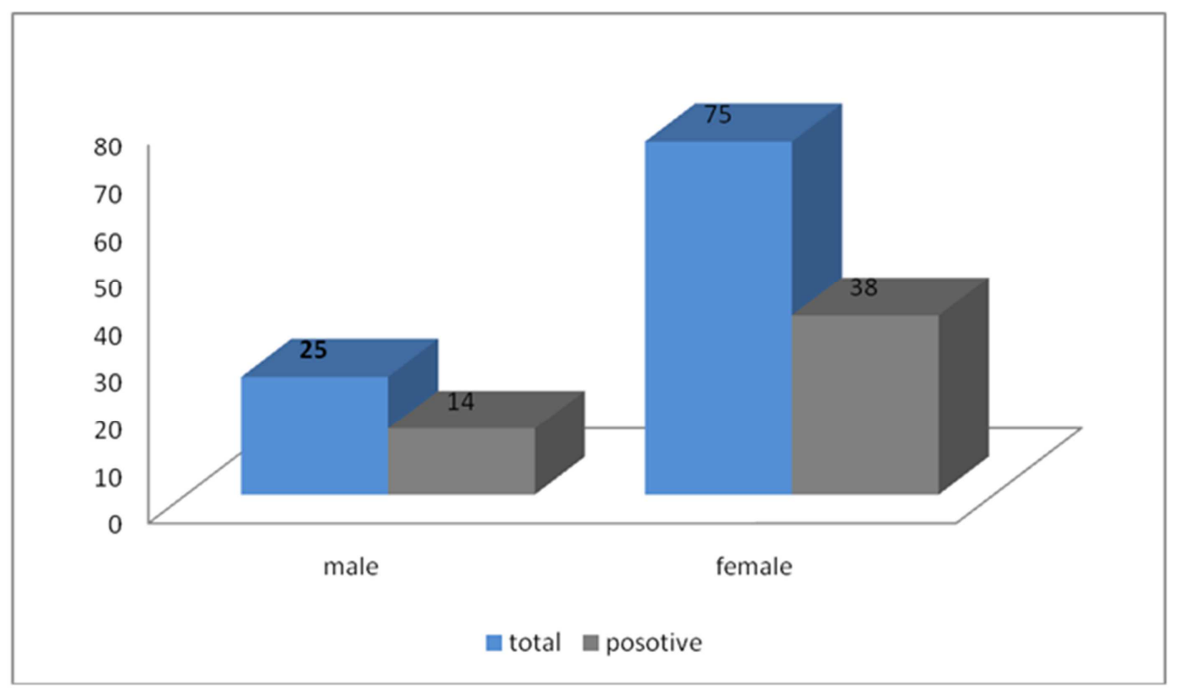

Figure 1. Distribution of specimen according to gender and the number of positive culture for each gender. 
Table 1. Significant and insignificant growth

\begin{tabular}{llll}
\hline Total Number of specimens & Significant growth (\%) & Insignificant growth(\%) \\
\hline \multirow{3}{*}{100 specimens } & $52 \%$ & LF $(\%)$ & $48 \%$ \\
& NLF $(\%)$ & 48 specimens $92.3 \%$ & \\
\hline
\end{tabular}

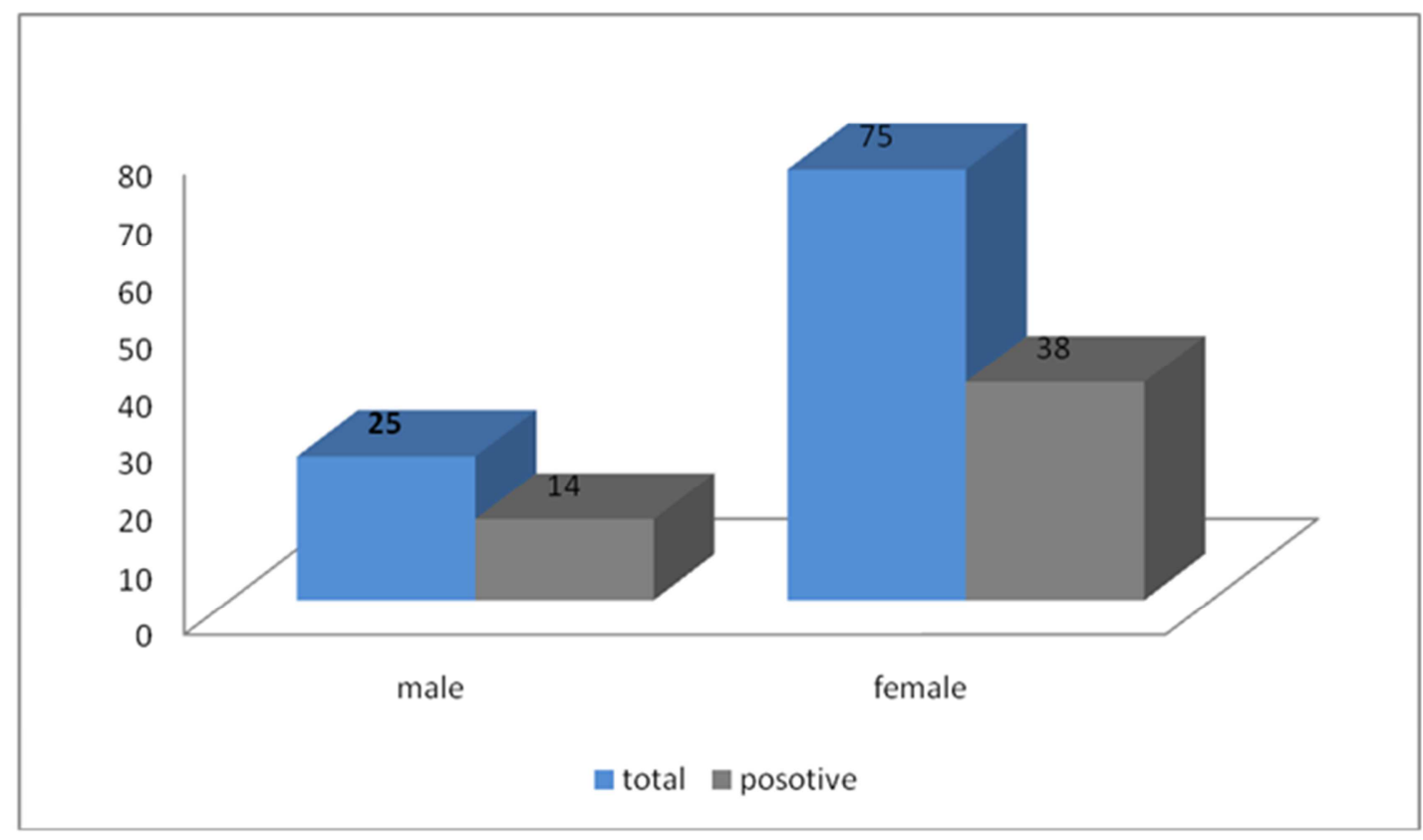

Figure 2. Distribution of specimen according to gender and the number of positive culture for each gender.

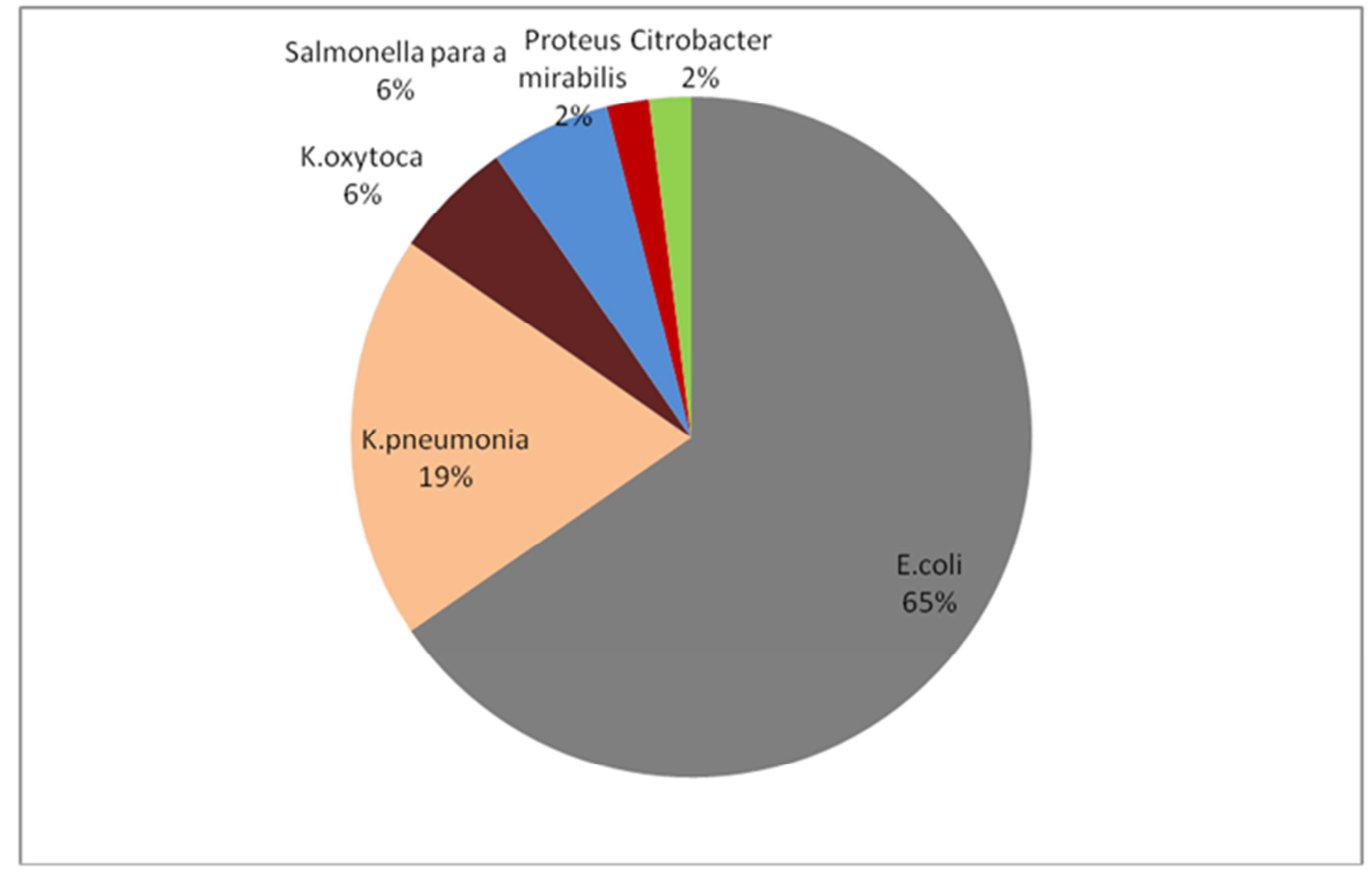

Figure 3. Percent ofeach isolated Enterobacteriaceae. 


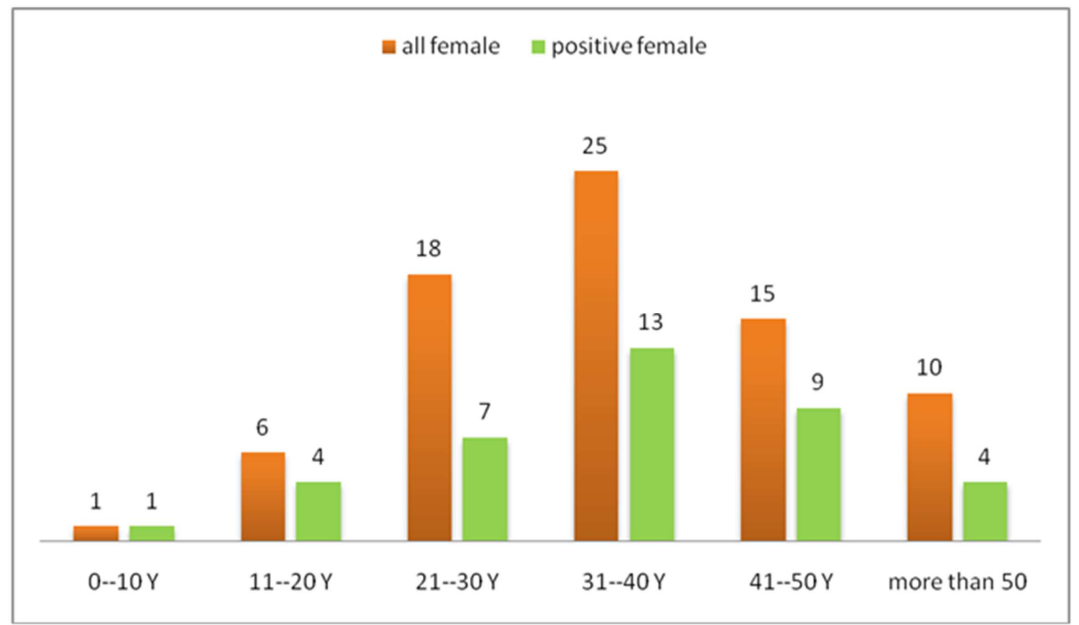

Figure 4. Distribution of female specimens according to ages andpositive culture.

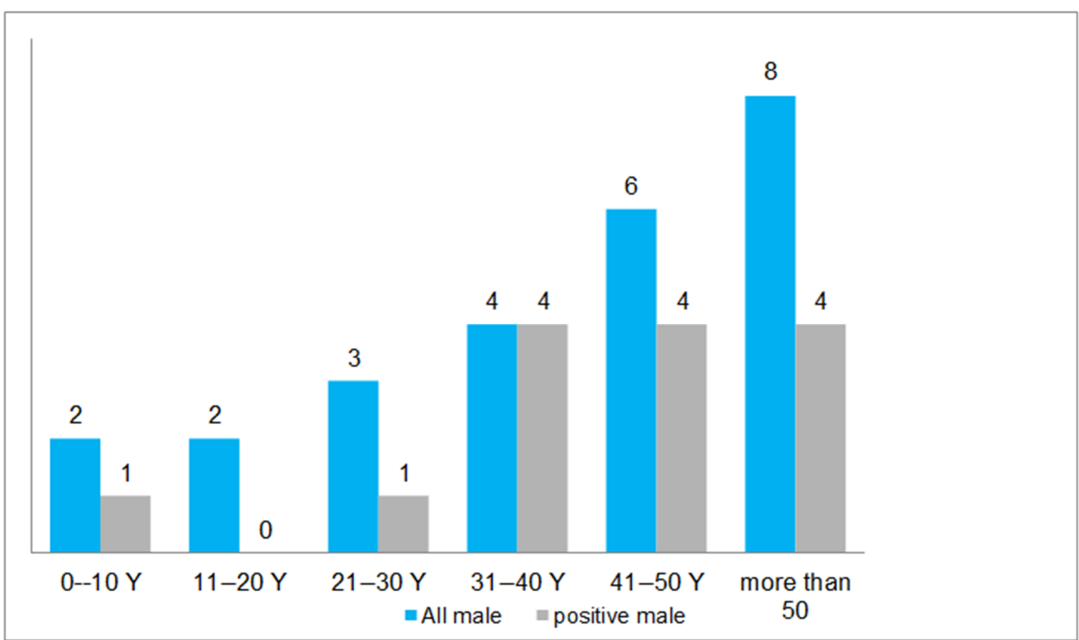

Figure 5. Distribution of male specimens according toages andpositive culture.

Table 2. Number and percentage of antimicrobial sensitivity tests( $S=$ sensitive, $R=$ resistance).

\begin{tabular}{|c|c|c|c|c|c|c|c|c|c|c|c|c|}
\hline \multicolumn{2}{|c|}{ Organisms } & AS & BA & CI & $\mathrm{CH}$ & PR & TE & $\mathrm{CP}$ & $\mathbf{A k}$ & SC & GF & $\mathbf{N X}$ \\
\hline \multirow{12}{*}{ S } & \multirow[b]{2}{*}{ E.coli } & 1 & 13 & 22 & 34 & 18 & 7 & 21 & 33 & 14 & 29 & 19 \\
\hline & & $3 \%$ & $38 \%$ & $65 \%$ & $100 \%$ & $53 \%$ & $20 \%$ & $62 \%$ & $97 \%$ & $41 \%$ & $85 \%$ & $55 \%$ \\
\hline & \multirow{2}{*}{ k.pnuemonie } & 10 & 4 & 4 & 9 & 1 & 7 & 6 & 7 & 4 & 8 & 5 \\
\hline & & $100 \%$ & $40 \%$ & $40 \%$ & $90 \%$ & $10 \%$ & $70 \%$ & $60 \%$ & $70 \%$ & $40 \%$ & $80 \%$ & $50 \%$ \\
\hline & \multirow{2}{*}{ k.oytoca } & 0 & 0 & 0 & 2 & 0 & 1 & 2 & 3 & 2 & 3 & 2 \\
\hline & & $0 \%$ & $0 \%$ & $0 \%$ & $67 \%$ & $0 \%$ & $33 \%$ & $67 \%$ & $100 \%$ & $67 \%$ & $100 \%$ & $67 \%$ \\
\hline & \multirow{2}{*}{ S.para A } & 0 & 3 & 3 & 3 & 2 & 3 & 3 & 3 & 2 & 2 & 3 \\
\hline & & $0 \%$ & $100 \%$ & $100 \%$ & $100 \%$ & $67 \%$ & $100 \%$ & $100 \%$ & $100 \%$ & $67 \%$ & $67 \%$ & $100 \%$ \\
\hline & \multirow{2}{*}{ Proteus } & 0 & 0 & 1 & 1 & 1 & 1 & 0 & 1 & 0 & 1 & 0 \\
\hline & & $0 \%$ & $0 \%$ & $100 \%$ & $100 \%$ & $100 \%$ & $100 \%$ & $0 \%$ & $100 \%$ & $0 \%$ & $100 \%$ & $0 \%$ \\
\hline & \multirow{2}{*}{ ciyrobacter } & 0 & 0 & 0 & 1 & 0 & 0 & 1 & 1 & 1 & 1 & 1 \\
\hline & & $0 \%$ & $0 \%$ & $0 \%$ & $100 \%$ & $0 \%$ & $0 \%$ & $100 \%$ & $100 \%$ & $100 \%$ & $100 \%$ & $100 \%$ \\
\hline \multirow{12}{*}{$\mathrm{R}$} & \multirow{2}{*}{ E.coli } & 33 & 21 & 12 & 0 & 16 & 27 & 13 & 1 & 20 & 5 & 15 \\
\hline & & $97 \%$ & $62 \%$ & $35 \%$ & $0 \%$ & $57 \%$ & $80 \%$ & $38 \%$ & $3 \%$ & $59 \%$ & $15 \%$ & $45 \%$ \\
\hline & \multirow{2}{*}{ k.pnuemonie } & 0 & 6 & 6 & 1 & 1 & 3 & 4 & 3 & 6 & 2 & 5 \\
\hline & & $0 \%$ & $60 \%$ & $60 \%$ & $10 \%$ & $90 \%$ & $30 \%$ & $40 \%$ & $30 \%$ & $60 \%$ & $20 \%$ & $50 \%$ \\
\hline & \multirow{2}{*}{ k.oytoca } & 3 & 3 & 3 & 1 & 3 & 2 & 1 & 0 & 1 & 0 & 1 \\
\hline & & $100 \%$ & $100 \%$ & $100 \%$ & $33 \%$ & $100 \%$ & $67 \%$ & $33 \%$ & $0 \%$ & $33 \%$ & $0 \%$ & $33 \%$ \\
\hline & \multirow{2}{*}{ S.para A } & 3 & 0 & 0 & 0 & 1 & 0 & 0 & 0 & 1 & 1 & 0 \\
\hline & & $100 \%$ & $0 \%$ & $0 \%$ & $0 \%$ & $33 \%$ & $0 \%$ & $0 \%$ & $0 \%$ & $33 \%$ & $33 \%$ & $0 \%$ \\
\hline & \multirow{2}{*}{ Proteus } & 1 & 1 & 0 & 0 & 0 & 0 & 1 & 0 & 1 & 0 & 1 \\
\hline & & $100 \%$ & $100 \%$ & $0 \%$ & $0 \%$ & $0 \%$ & $0 \%$ & $100 \%$ & $0 \%$ & $100 \%$ & $0 \%$ & $100 \%$ \\
\hline & \multirow{2}{*}{ ciyrobacter } & 1 & 1 & 1 & 0 & 1 & 1 & 0 & 0 & 0 & 0 & 0 \\
\hline & & $100 \%$ & $100 \%$ & $100 \%$ & $0 \%$ & $100 \%$ & $100 \%$ & $0 \%$ & $0 \%$ & $0 \%$ & $0 \%$ & $0 \%$ \\
\hline
\end{tabular}




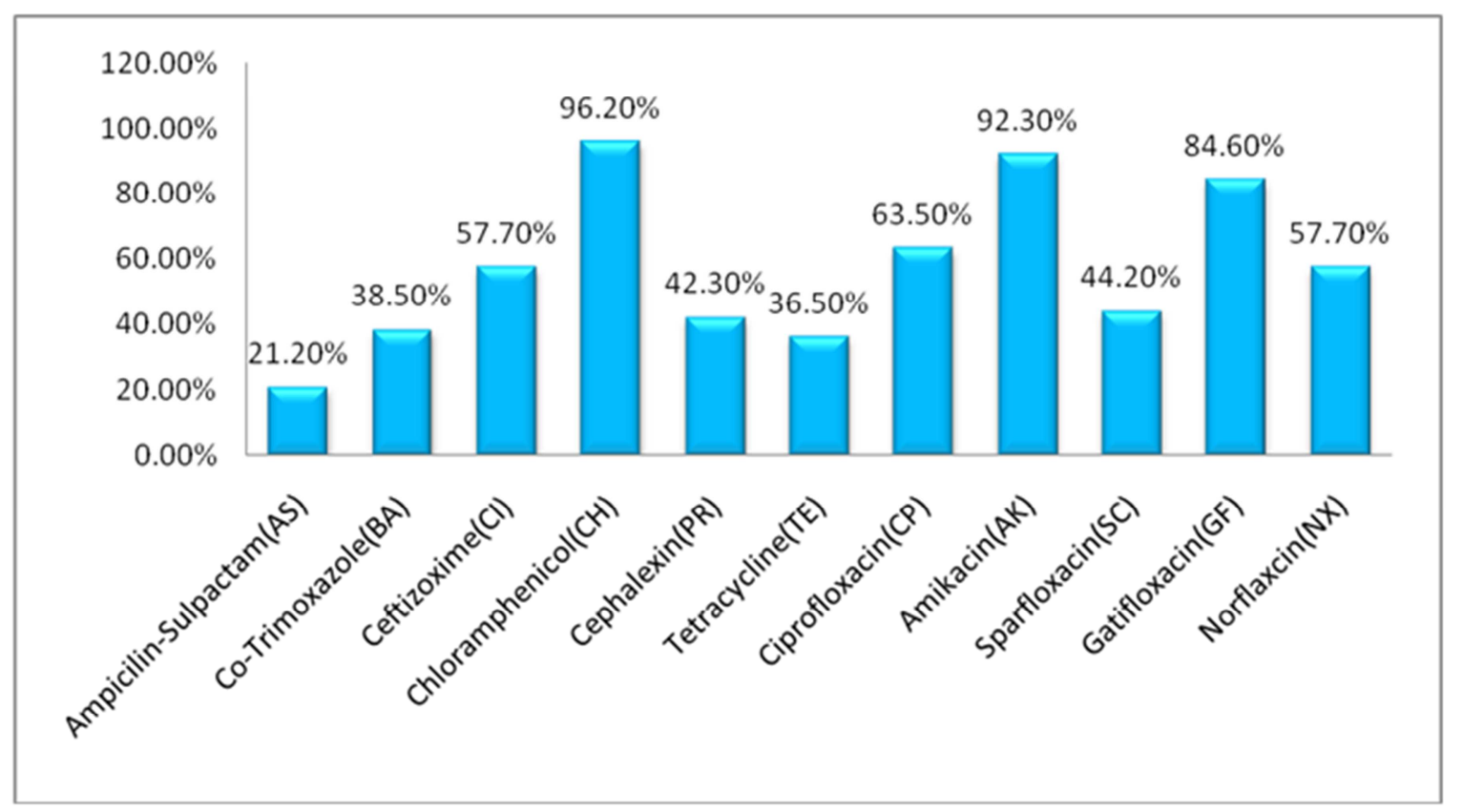

Figure 6. Percentage of each antimicrobial used for UTI infections.

\section{Discussion}

This study was conducted to determine the main Enterobacteriaceae caused infection in community acquired UTI patient and the antimicrobials sensitivity test. The results revealed that the lactose ferment Enterobacteriaceae type represent $(92 \%)$, while non lactose fermented organisms represent $(8 \%)$ and this result agreed withthe result obtained by Panahi Y., et al. [4]. The ratio between male to female is $1: 3,25 \%--75 \%$ respectively this result is nearly similar to result 1:2 report by Sharifian et al. [5].

The result showed that UTI cases among female of age group (31-40 years) were found more susceptible to UTI $(33.4 \%)$, that agree with results obtained by Orret and Shurland [6]. In the present study the most prevalent organisms were E.coli (65.4\%) of all Enterobacteriaceae isolated, this result agree that obtained by Ekweozor and Onyemenen [7], who showed that E.coli account (62\%), the followed organism is Klebsiella Spp this result is similar to that obtained by Gupta K., et al. [8]. In our study the elder males (over 50 years) are more susceptible to UTI this result agree with a result obtained by Torkaman M., et al [9]. In the present study the anti microbial sensitivity test demonstrated that AmpicillinSulbactam $(21.2 \%)$ and Tetracycline $(36.5 \%)$ are the lowest active agent, While Chloramphenicol (96.2\%) and Amikacin (92.3), are the highest active agent this result confirmed the resultobtained by Modarres et al, [7].

On other hand, the result showed that E.coli was more susceptible to Chloramphenicol $(100 \%$,), Amikacin $(97 \%)$ and Gatifloxacin (97\%), respectively, this results disagree with a result obtained by (Sharifian et al [5]. who found that E. coli was most sensitive toCeftizoxime.

\section{Conclusions and Recommendations}

\subsection{Conclusions}

I. The most prevalent organism in UTI infections is E.coli.

II. Female are more susceptible to UTI than male.

III. Ages from 31-40 are the most infected among female.

IV. Men whom ages more than 50 are more susceptible to UTI than other ages.

V. Chloramphenicol and Amikacinare the highest active agents in most strains.

VI. Ampicillin-Sulpactam is the lowest active antimicrobial agents due to high resistant rate.

VII. Resistance rate of Enterobacteriaceae increased to commonly used antimicrobial agents.

\subsection{Recommendations}

a. Urine specimen should investigated for culture and susceptibility test before giving the patient any therapy to decrease the resistant rate among organisms causing community acquired UTI.

b. increase awarenessabout thehazards of using antibiotic for treatment without pre -therapy culture and how bacteria gain resistant among people with UTI infection.

c. Uses of API $20 \mathrm{E}$ are recommended for investigation of all Enterobacteriaceae in Microbiology lab for more accuracy.

d. Establishment of antimicrobial policies and treatment guidelines.

e. Uses of Chloramphinicol and Amikacin as routine treatment for community acquired UTI infections.

f. In depth studies by using modified technique (molecular methods) for precision and accuracy is recommended. 


\section{References}

[1] Okesola. A. O and Makanjuola. O. (2009). Resistance to Third -generation Cephalosporins and other Antibiotics by Enterobactericeae in Western Nigeria, American Journal of Infection disease, 5(1):17-20.

[2] Murray Patrick. R, Rosenthal. Kens, Kabayashi George S and Pfaaller. Michael A. (2002). Medical Microbiology, 4th edition, Mosby. P266-279.

[3] Rayan. Kenneth. J and George. C. Ray. (2004). Sherris Medical Microbiology4 th edition, McGraw -HILL, New York, Chicago. P. 343-367.

[4] Panahi Y, Beiraghdar F, Moharamzad Y, Matinzadeh ZK, EinollahiB. The incidence of urinary tract infections in febrile children during a two-year period in Tehran, Iran. Trop Doct. 2008; 38(4):247-9.

[5] Sharifian M, Karimi A, Tabatabaei SR, Anvaripour N.

Microbial sensitivity pattern in urinary tract infections in children: a single center experience of 1,177 urine cultures. Jpn J Infect Dis. 2006;59(6):380-2.

[6] Orret and Shurland, 1998. Bacterial etiologic agents of urinry tract infection in Hospitalized patients Nigeria, Abuja.

[7] Modarres S, Oskoii NN. Bacterial etiologic agents of urinary tract infection in children in the Islamic Republic of Iran. East MediterrHealth. 1997;3:290-5.

[8] Gupta K. Emerging antibiotic resistance in urinary tract pathogens Infect Dis Clin North Am. 2003;17(2):243-59.

[9] Torkaman M, Afsharpaiman SH, Hoseini MJ, Moradi M, Mazraati A, Amirsalari S, et al. Platelet count and neonatal sepsis: a high prevalence of Enterobacter spp. Singapore Med J. 2009; 50(5):482-512

[10] Collee, J. G; Morinon, B. P.; Fraser, A. G. Simmons, A. (1996). Maki and McCartney Practical Medical Microbiology (14th edition). Churchill Livingstone New York. 